\title{
Simulation of the capillary action via FlowVision software
}

\author{
Lenar Ishbulatov ${ }^{1, *}$, Sergey Galyshev ${ }^{1}$, Pavel Solovev ${ }^{1}$, and Andrey Aksenov ${ }^{2}$ \\ ${ }^{1}$ FSBEE USATU, Department of Materials Science and Physics of Metals, 450008, Ufa, Russia \\ ${ }^{2}$ JIHT of RAS, Laboratory "Program systems", 125412, Moscow, Russia
}

\begin{abstract}
The paper considers the simulation of fluid flow processes in the FlowVision software with taking into account the capillary action, wettability, surface tension forces and fluid viscosity. Test problems were solved to take into account the influence of surface wettability on the basis of modeling a drop of mercury placed on a hydrophobic surface, as well as taking into account the capillary action by immersing a thin tube in a liquid and raising its level. Qualitative and quantitative analysis of the results was made, conclusions were made about the applicability of the FlowVision software for simulating the process of impregnating the framework of reinforcing fibers with a binder when forming products from metal matrix composites.
\end{abstract}

\section{Introduction}

Composite materials $(\mathrm{CM})$ due to their unique physical\&mechanical properties, such as high specific strength and stiffness, low weight, increased resistance to fatigue failure and significant impact toughness, have found wide application in most knowledge-intensive industries: aircraft and rocketry, automotive, shipbuilding, etc.

In the world practice composites on the polymer matrix have found the greatest application at the present time because of the relatively low density (compared with the metals and alloys traditionally used) and good adhesion over the matrix-fiber interface.

However, composites on a metal matrix also have a number of advantages over polymer composites: increased strength and rigidity in directions perpendicular to reinforcement, significantly higher heat resistance, high temperature strength and an extended operating temperature range. These advantages make it important to use metal matrix composites in the construction of highly loaded (both mechanically and thermally) units and aggregates of aircraft, such as, for example, aircraft engines. At the same time, CMs on the metal matrix have significantly less weight compared to titanium and nickel alloys, currently used in the "hot" part of aviation GTE.

Nevertheless, there are a number of technological aspects that make it difficult to use metal matrix CMs in the aircraft structural elements mass production: the problems of protecting reinforcing fibers from damage by matrix material during composite molding, the problem of the fibers wettability by the melt of the matrix, questions regarding the

${ }^{*}$ Corresponding author: blacksail772@mail.ru 
choice of technological modes for pouring or forming composites, providing the best reinforcing cage impregnation, minimizing the number of pores and increasing the mechanical characteristics of metal matrix CM.

To solve most of the above issues, modern modeling techniques and software products such as ANSYS Multiphysics, PAM-RTM, FlowVision, etc. can be used. The simulation results allow to significantly reduce the time of product finishing, to obtain technological parameters ensuring product high quality, and to reduce its prime cost increasing the manufacturer competitiveness.

Let's analyze the capabilities of FlowVision software. It is designed to simulate the gasand hydrodynamics of viscous liquids and gases flow processes with taking into account wettability effects, surface tension forces, hydraulic resistance, etc. The advantages of this software are relatively low requirements for computer resources and relatively low cost in comparison to major world competitors such as ANSYS, PAM-RTM, ProCAST, etc.

To confirm the suitability of the considered program for solving problems related to the reinforcing fibers framework impregnation, a number of test problems were worked out that demonstrating the coincidence of simulation results and real physical phenomena.

\section{Theoretical foundations of liquids flow modeling}

Modeling of non-stationary fluid motion in computational domains with moving boundaries, as well as modeling of contact interfaces (free surface) is an integral part of modern problems of computational fluid dynamics. In the FlowVision software (FV) the most universal method for calculating such boundaries based on Eulerian calculated walls is used. The calculation package uses automatic calculating mesh generation, dynamically adapting to the solution process, which also leads to a change in the grid topological structure and its size [1].

When mathematical modeling of transport processes of immiscible phases, in which the behavior of interphase surfaces plays a significant role, is processed, it is necessary to take into account capillary actions.

The basis of the question of the liquids behavior in the vessels is such physical processes as surface tension and wetting [2]. Capillary phenomena due to them are studied in complex. Under the influence of the surface tension force, the wetting liquid in the capillaries is above the level at which it should be in accordance with the law of the communicating vessels. Conversely, the non-wetting substance is located below this level (see Fig. 1).

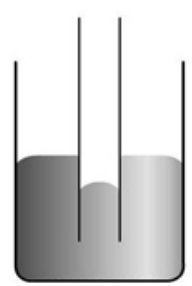

a)

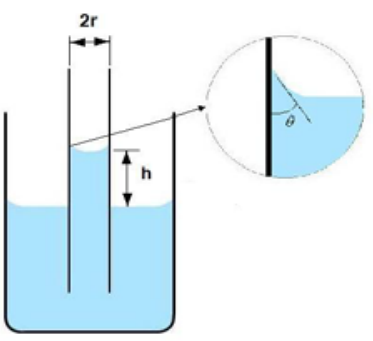

b)

Fig. 1. Behavior of the liquid in contact with a) non-wetting and b) wetting surfaces.

The moment of stopping the water ascension in a narrow tube occurs when the gravitational force of the substance is equal to the surface tension F. This moment determines the height of the liquid ascension. Capillary phenomena are caused by two 
oppositely directed forces: gravity $\mathrm{G}$ causes the liquid to fall down; surface tension $\mathrm{F}$ moves the water upwards.

\subsection{Features of modeling in FlowVision}

The following initial assumptions are used in the FV software product:

1. Mixing path - a smooth profile of turbulent viscosity is determined by the classical Prandtl expression.

2. Gradientless and detachable experimental velocity profiles superposition.

3 . Boundary conditions of the third kind for the momentum equation.

4. Roughness allowance by replacing molecular viscosity with an effective [3].

The basic idea of the finite element method is that any continuous quantity such as temperature, pressure and displacement can be approximated by a discrete model that is built on the set of piecewise continuous functions defined on a finite number of subdomains. The accuracy of the calculation depends on the number of finite elements that describe the model, and is limited only by the processing power of the computer [4].

The software package «FlowVision-HPC» is based on a finite-dimensional Cartesian computational grid. Grid local dynamic adaptation is performed in accordance with userdefined criteria. Initially, the grid consists of rectangular cells, but near the boundary of the calculated area, there is a Boolean subtraction of non-calculated volumes from rectangular cells and the formation of arbitrary calculated polyhedra. Equations of fluid motion are solved by means of an implicit splitting scheme. Algebraic equations arising in the equations approximation of fluid motion, energy, contact surface transfer, turbulent characteristics transfer, etc., are solved by methods such as Krylov's subspaces [3].

\section{Computational experiment}

This paper is devoted to modeling the capillary action with taking into account the wettability, surface tension forces and viscosity of the medium.

\subsection{Modeling the wetting process}

The task of identifying the effect of wettability is based on modeling the mercury drop formation on a hydrophobic surface.

In Fig. 2a is shown the finite element model of a mercury drop.

As initial data, mercury parameters such as molar mass, density, viscosity, surface tension were used. The wetting angle over the reference surface was set at 175 degrees. The external environment is air having standard atmospheric parameters - pressure $101325 \mathrm{~Pa}$, temperature $20^{\circ} \mathrm{C}$.

After the modeling process, the appearance of the mercury drop model (Fig. 2b), qualitatively similar to the real drop (Fig. 2c).

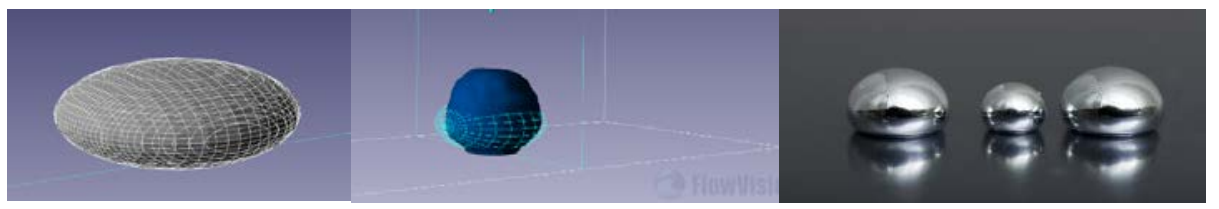

a)

b)

c)

Fig.2. Modeling of a mercury drop a) the finite element model of the wettability effect b) a model calculated in FV c) real drops of mercury on a non-wettable surface. 


\subsection{Modeling the capillary effect}

The problem of modeling the capillary action concluded in comparison the change of liquid (water) column height in the tube obtained in FV with theoretically predicted by the Borelli-Jurin formula. The simulation conditions are the same as those indicated in the previous section.

Initial data: a tube $3 \mathrm{~mm}$ in diameter with a wetting angle of 5 degrees, a water surface tension force $(0.073 \mathrm{~N} / \mathrm{m})$, a water density of $1000 \mathrm{~kg} / \mathrm{m}^{3}$, a wetting angle of 5 degrees, an initial liquid height of $3 \mathrm{~mm}$, a tube entering the water for $2 \mathrm{~mm}$. The density of the medium (air) is determined programmatically by the law of an ideal gas, the viscosity of the liquid (water) is $0.001 \mathrm{kgf} /(\mathrm{m} * \mathrm{~s})$.

To find the height of the liquid, we use Juren's formula:

$$
h=\frac{2 \sigma \cdot \cos \Theta}{r\left(\rho-\rho_{0}\right) g}
$$

where $r$ is the tube radius;

$\rho$ is the liquid density;

$\rho_{0}$ is the external medium (air) density;

$\Theta$ is the wetting angle;

$\sigma$ is the surface tension force. $10 \mathrm{~mm}$.

The theoretical value of the liquid column height, calculated by the above formula, was

Fig. 3 shows the finite element model used to simulate the capillary action.

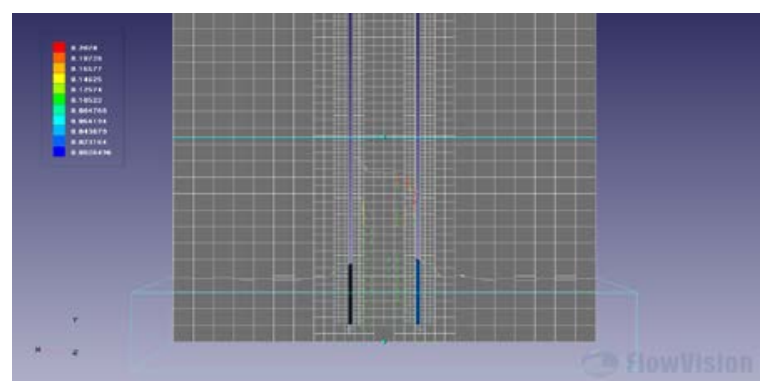

Fig.3. Finite element model of the capillary action.

The spatial model is divided into components, and the calculation occurs in each part separately, then all calculations within one step are summed.

Problems with a free surface must be solved with a fine grid and a smaller time step in order to obtain more accurate results. For this, the "adaptation" function was used. With this function, the grid is grinded nearby the tube wall, which increases the accuracy of calculations.

Figure 4 shows the results of fluid movement due to the capillary action manifestation. The patterns of fluid distribution and the motion velocity vectors are presented.

It can be seen from the figure that the liquid column reaches a maximum height of $13.5 \mathrm{~mm}$ after a time of 0.1 seconds from the calculation beginning, i.e. the water rose $10.5 \mathrm{~mm}$ above the original level. Further, the oscillatory processes in the system characterize the establishment of a gravity and surface tension forces balance. 


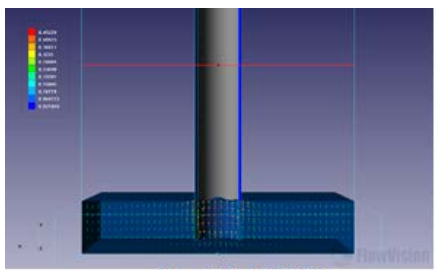

Step № 1 - 0,00025 s.

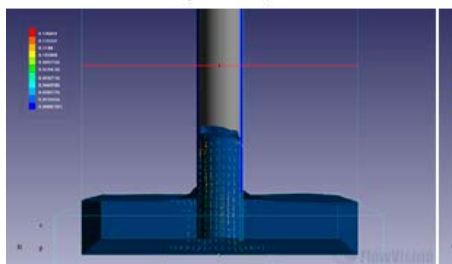

Step № $900-0,225$ s.

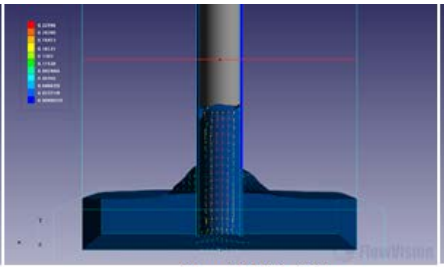

Step № $200-\mathbf{0 , 0 5}$ s.

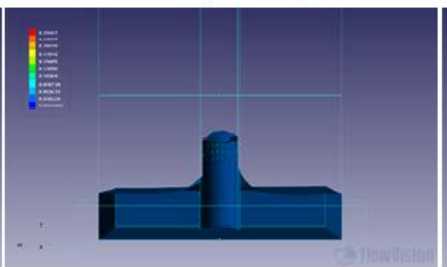

Step № $1200-0,3$ s.

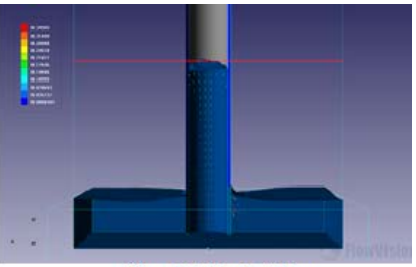

Step № $500-0,125$ s.

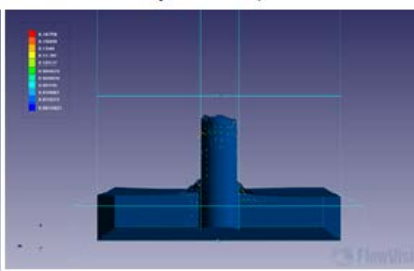

Step № $1500-0,375 s$.

Fig. 4. Movement of fluid due to the capillary action manifestation.

After a time of 0.4 seconds, the final equilibration occurs and the liquid column height stops at a value of $10 \mathrm{~mm}$. Subsequent calculations (up to $4 \mathrm{~s}$ ) have shown that there is no further change in the height of the liquid column (see Figure 5).

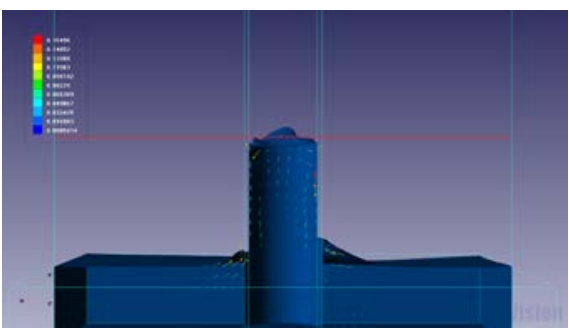

Fig. 5. Calculation step No. 15000 - 3.75 seconds.

It can be seen from Fig. 5 that the fluid velocity vectors are oriented chaotically, which indicates that there is no liquid directional movement relative to the tube.

Fig. 6 is a graph of the change in the maximum fluid velocity and the liquid column height versus time since the process start.

The graph shows two speed peaks that correspond to an active change in the height of the liquid column. The speed in this case exceeded the value of $0.25 \mathrm{~m} / \mathrm{s}$. The rate of chaotic motion velocity of the equilibrated liquid was $0.2 \mathrm{~m} / \mathrm{s}$. The trend line of the velocity change is monotonically decreases, which indicates a gradual relaxation of the system and the absence of any significant changes.

The maximum height of the liquid is reached after $100 \mathrm{~ms}$ and is balanced after $400 \mathrm{~ms}$, as it was said before.

According to the results obtained, the height of the column of liquid should be equal to $10 \mathrm{~mm}$, but according to simulation calculations, the height of the column was balanced at the level of $7 \mathrm{~mm}$. The discrepancy was $30 \%$. 


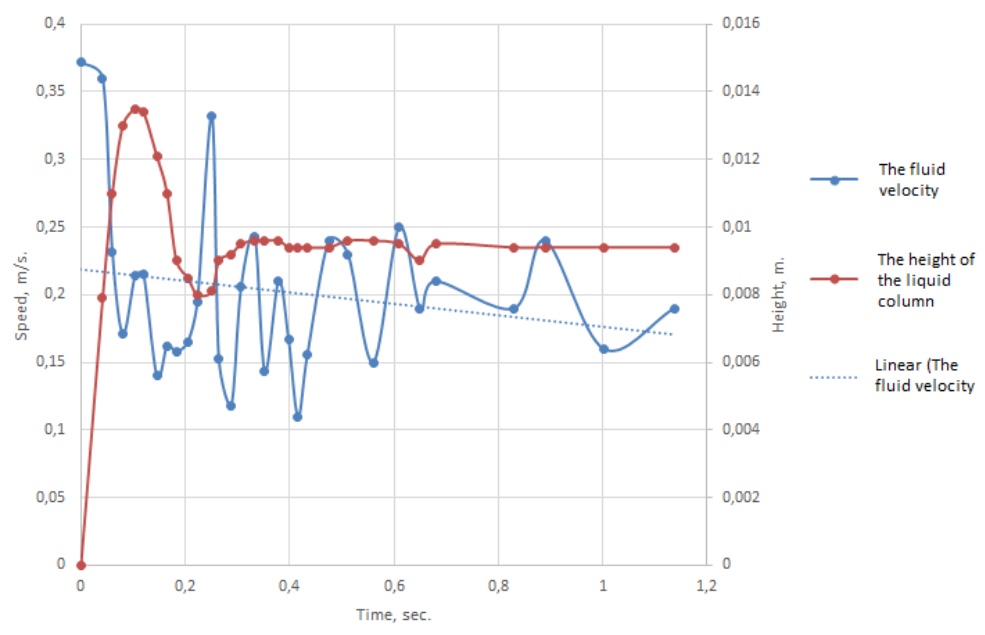

Fig. 6. The graph of the change in the fluid maximum velocity and the liquid column height versus time from the process beginning.

\section{Conclusion}

The obtained results confirm the possibility of the FlowVision software to take into account the capillary action. Qualitative patterns of fluid distribution in both problems correspond to the real state of affairs.

Comparison of simulation results and theoretical calculations showed that the quantitative discrepancy in the data was about $30 \%$. This may be due to the constraints imposed on the Borelli-Jurin formula: the surface of the meniscus is a sphere, the height of the liquid raising (lowering) is much greater than the radius of the capillary. To solve this problem, it may be necessary to introduce some corrective coefficients in the simulation.

In general, the simulation results confirm the suitability of the FlowVision software product for simulating fluid flow processes in the given geometry space, taking into account the wettability, surface tension forces, viscosity, capillary action, etc. This makes possible to solve the problem of impregnating reinforcing fibers with a binder when forming metal matrix composites in a FlowVision calculation package.

\section{References}

1. A.A. Aksenov, A.A. Dydkin, S.A. Harchenko, Investigation of the calculation parallelization efficiency of moving bodies and free surfaces motion in FlowVision on computers with distributed memory, Vichislitelnie metodi и programmirovanie, T. 10, pp. 132-140 (2009)

2. K.A. Potashev, N.D. Yakimov, I.P. Breus Transport processes in porous medium: capillary actions, VANT, Matematicheskoy vodelirovanie fizicheskih prosesov, v. 3, pp. $72-79$ (2005)

3. S.V. Jluktov, A.A. Aksenov, S.A. Harchenko, I.V. Moskalev, G.B. Sushko, A.S Shishaeva, Simulation of detached flows in the FlowVision-HPC software, Vichislitelnie metodi и programmirovanie, T. 11, pp. 234-245 (2010)

4. L. Segerlind, The application of the finite element method (Mir, 1979) 